\title{
Diabetes and HIV
}

\author{
Author: John Quin ${ }^{\mathrm{A}}$
}

The co-existence of diabetes mellitus and HIV infection poses significant challenges for both patient and physician. This article reviews the clinical problems, the implications for treatment plans and potential confusions that can arise when managing patients who have both conditions.

As a doctor I can tell you that, medically speaking, I'd rather have HIV than diabetes. Dr Max Pemberton, The Spectator, April 2014

\section{Introduction}

Dr Pemberton's quote above surprised many but reminds us that HIV-related mortality and morbidity have significantly declined since the widespread use of highly active antiretroviral therapy (HAART). ${ }^{1}$ Pemberton recalls the 'sense of crushing dread' that physicians and the public felt during the early 1980s when faced with the, then incurable, catastrophe that was AIDS. I recall my own first referral to see someone with HIV who had developed diabetes. How to approach and communicate this new diagnosis? Here's the wrong way: 'Well, maybe you don't need to worry about diabetes because...'

Times change. Today it is the concomitant diagnosis of diabetes that is to be feared in those with HIV. This double burden is a significant challenge for patients and carers. Cardiovascular complications and death due to these are now the more pressing challenge for HIV physicians and diabetologists. The DAD study has suggested that the incidence of diabetes increases with cumulative exposure to combination HAART. ${ }^{2}$ This paper reviews the impact of diabetes on those with HIV and argues for subspecialty-specific clinics to help care for these patients.

\section{Changes in glucose homoeostasis in HIV}

Insulin resistance, as opposed to insulin deficiency, is the main pathogenic factor in the development of diabetes in patients with HIV infection. Concomitant hepatitis C infection may also play a role. ${ }^{3}$ Growth hormone deficiency can be seen in HIV-infected patients and this too may be

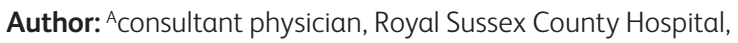
Brighton, UK a factor in the development of insulin resistance along with increased accumulation of visceral adipose tissue. ${ }^{4}$ The virus itself may also be implicated in pancreatic dysfunction. ${ }^{5}$

\section{Changes associated with HIV therapy}

Type 2 diabetes mellitus appears to be four times more common in HIV-infected men exposed to HAART compared with an HIV-negative cohort. ${ }^{6}$ The protease inhibitors or PIs (eg atazanvir, darunavir and ritonavir) have been shown to increase insulin resistance, reduce insulin secretion and interfere with glucose transporter type 4-mediated glucose transport. ${ }^{7}$ The PIs have also been shown to interfere with cellular retinoic acid-binding protein type 1 (CRAB-1) which interacts with the peroxisomal proliferator-activated receptor $\gamma(\operatorname{PPAR} \gamma)$. The inhibition of PPAR $\gamma$ leads to insulin resistance and release of free fatty acids. ${ }^{8}$ A reduction in first phase insulin release has been noted with some forms of HAART. ${ }^{9}$ Some nucleoside reverse transcriptase inhibitors are thought to cause metabolic abnormalities and increase the risk of diabetes, ${ }^{2}$ and combination therapy may also be an additional risk factor for its development. ${ }^{10}$ Further work is likely to help identify the individual propensities of drugs to cause these effects. There is thus the likelihood that future therapeutic regimens may need to be tailored to reduce metabolic derangement. The risks currently seem to be highest with stavudine. Other proposed abnormalities include alteration in leptin/adiponectin dynamics and mitochondrial dysfunction. ${ }^{11}$ Clearly these concerns do not mean the exclusion of HAART in patients with HIV, but awareness of the development of diabetes may lead to pharmacological 'tweaking' of management.

\section{HIV lipodystrophy syndrome}

The development of insulin resistance, hyperglycaemia and diabetes mellitus has also been linked to the HIV lipodystrophy syndrome. ${ }^{12}$ The aetiology of this condition is unknown. Most patients thought to have the syndrome have central, visceral obesity with peripheral fat wasting.

\section{Screening}

A reasonable case can be made for screening for diabetes mellitus in all newly presenting patients with HIV, and at 3 and 6 months after starting HAART. Equivocal results will necessitate follow-up with oral glucose tolerance testing. As yet 
there is no strong argument for using glycated haemoglobin as a screening tool in this situation.

\section{Treatment}

As per guidelines for the management of type 2 diabetes mellitus, there must be robust attempts to modify risk factors for cardiovascular disease (CVD). Dietary measures, exercise, smoking cessation, antihypertensives and lipid-lowering agents are often indicated. Simvastatin is contraindicated because of its interaction with the cytochrome $\mathrm{P} 450$ system. ${ }^{13}$ Rigorous screening and treatment for other concomitant infections (hepatitis C, syphilis, balanitis and vulval candidiasis) are warranted. Ease of access to dietetic, podiatry, retinal screening and diabetes nurse specialist services all argue for a joint HIV/ diabetes service.

Drug treatment to allay hyperglycaemia is similar to that used in the non-HIV population with the following caveats.

> Metformin: this can reduce insulin resistance in HIV patients. ${ }^{14}$ The risks of lactic acidosis remain rare but HIV physicians try to avoid HAART combinations that, in theory, could exacerbate this possibility. Diarrhoea and faecal incontinence are well recorded with metformin and so the drug may be inappropriate in HIV patients with coexistent gastrointestinal pathology.

> Thiazolidinediones: theoretically these drugs could be of benefit in those with lipodystrophy, given the known effects that they have on promoting adipocyte differentiation; however, clinical reports have been disappointing. ${ }^{15}$

\section{Psychological challenge/education}

Acknowledgement of these twin burdens as a significant challenge for the patient is key to the educational approach. We must not underestimate the psychological impact of a second diagnosis with serious prognostic import. Pill burden can be a significant issue. Depression is common in patients with both conditions and should be treated accordingly.

\section{Key points}

Mortality from HIV has significantly improved but patients with coexisting diabetes face major health challenges

Some forms of antiretroviral treatments may be associated with insulin resistance and pharmacological vigilance is required by carers

There is a rise in incidence of type 2 diabetes in the HIV population

Optimal treatment to reduce CVD risk can be provided at subspecialty diabetes/HIV clinics

KEYWORDS: diabetes mellitus, HIV, antiretroviral therapy, insulin resistance

\section{Confusions}

The following scenarios should be considered.

$>$ Night sweats: these may be due to nocturnal hypoglycaemia if over-insulinised. Alternatively it may be a sign of occult infection as a result of immunodeficiency. In particular tuberculosis must be considered and excluded.

$>$ Retinal changes: diabetic retinopathy must be distinguished from cytomegalovirus retinitis.

> Weight loss: this may be due to poor diabetes control or poor control of viral load. Tragically it is thought that, in Africa, many cases of type 1 diabetes are misdiagnosed as HIV. Health inequalities mean that, scandalously, all too often neither condition is treated appropriately.

$>$ Drug interactions: as highlighted earlier by choice of statin use. There are many other potential interactions of HAART and drugs used in patients with diabetes and CVD.

\section{Lessons from the politics of HIV services}

Services for HIV in the UK have benefited enormously from the sustained campaigns of informed patients and carers. There are clear lessons to be learned from this experience for those designing services for people with diabetes. Persistent lobbying and rigorous outlining of the threat that both conditions have to public health are necessary.

\section{References}

1 Palella FJ Jr, Delaney KM, Moorman AC et al. HIV Outpatient Study Investigators. Declining morbidity and mortality among patients with advanced immunodeficiency virus infection. $N$ Engl $J$ Med 1998;338:853-60.

2 De Wit S, Sabin CA, Weber R et al. Incidence and risk factors for new-onset diabetes in HIV-infected patients. The data collection on adverse events of anti-HIV drugs (D:A:D). Diab Care 2008;31:1224-9.

3 El-Zayadi A, Anis M. Hepatitis C virus induced insulin resistance impairs response to anti-viral therapy. World J Gastroenterol 2012;18:212-24.

4 Stanley TL, Grinspoon SK. GH/GHRH axis in HIV lipodystrophy. Pituitary 2009;12:143-52.

5 Dube MP. Disorders of glucose metabolism in patients infected with human immunodeficiency virus. Clin Infect Dis 2000;31:1467-75.

6 Brown TT, Cole SR, Xiuhong Li et al. Anitretroviral therapy and the prevalence and incidence of diabetes in a multicenter AIDS Cohort study. Arch Intern Med 2005;165:1179-84.

7 Hertel J, Struthers H, Horj CB, Hruz PW. A structural basis for the acute effects of HIV protease inhibitors on GLUT4 intrinsic activity. J Biol Chem 2004;279:55147-52.

8 Lee GA, Rao M, Greenfeld C. The effects of HIV protease inhibitors on carbohydrate and lipid metabolism. Curr Infect Dis Respir 2004; 6:471-82.

9 Woerle HJ, Mariuz PR, Meyer C et al. Mechanisms for the deterioration in glucose tolerance associated with HIV protease inhibitor regimens. Diabetes 2003;52:918-25.

10 Fleishman A, Johnsen S, Systrom DM et al. Effects of a nucleoside reverse transcriptase inhibitor, stavudine, on glucose disposal and mitochondrial function in muscle of healthy adults. Am J Physiol Endocrinol Metab 2007;292:E1666-73.

11 Samaras K, Wand H, Law M, Emery S, Cooper D, Carr A. Prevalence of metabolic syndrome in HIV-infected patients receiving highly active retroviral therapy using International 
Diabetes Federation and Adult Treatment Panel III criteria. Diab Care 2007;30:113-5.

12 Carr A, Samaras K, Chisholm D et al. Pathogenesis of HIV-1 protease inhibitor-associated peripheral lipodystrophy, hyperlipidaemia and insulin resistance. Lancet 1998;352:1881-3.

13 Fichtenbaum CJ, Gerber JG. Interactions between antiretroviral drugs and drugs used for the therapy of the metabolic complications encountered during HIV infection. Clin Pharmacokinet 2002;41:1195-211.

14 Hadigan C, Corcoran C, Basgoz N, Davis B, Sax P, Grinspoon G. Metformin in the treatment of HIV lipodystrophy syndrome. JAMA 2000;284:472-7.
15 Gelato MC, Mynarcik DC, Quick JL et al. Improves insulin sensitivity and body fat distribution in HIV-infected patients treated with rosiglitazone: a pilot study. J Acquir Immune Defic Syndr 2002;31:163-70.

Address for correspondence: Dr J Quin, Consultant physician, Royal Sussex County Hospital, Brighton BN2 5BE, UK. Email: john.quin@bsuh.nhs.uk

\title{
Diabetes and mental health
}

\author{
Authors: Chris Garrett ${ }^{A}$ and Anne Doherty ${ }^{B}$
}

\begin{abstract}
Diabetes is an increasingly common health problem, and accounts for one-tenth of NHS spending, chiefly managing avoidable complications. Approximately one-third of people with diabetes have psychological and/or social problems which impede their ability to self-manage their diabetes. Identifying certain indicators which suggest high risk of co-morbid mental health problems will allow these to be identified and treated early. Ensuring that any mental health problems are treated and social needs are met, will be valuable in improving the individuals health. Addressing the psychiatric and psychological barriers to good glucose control can help to reduce the burden of diabetes and its complications, on both the individual and the wider health service.
\end{abstract}

\section{Introduction}

Diabetes is an increasingly common health problem, especially in the West, where there is an emerging epidemic of type 2 diabetes. Diabetes accounts for one-tenth of NHS spending, and $80 \%$ of this is spent on managing potentially avoidable complications.

Many people who have diabetes struggle to optimise their diabetes control, often because of comorbid mental illness or psychological and social problems. Unfortunately, poor diabetes control has significant consequences for the individual, and if not addressed, will result in the development of complications that include blindness, kidney failure and even amputations. In addition to the consequences for the individual, these complications have an impact on health services in the form of

Authors: Aclinical research fellow, Institute of Psychiatry, King's College London, UK; ${ }^{B}$ Consultant liaison psychiatrist, King's College Hospital, London, London, UK increased admissions, out-patient attendances and emergency department presentations with diabetes-related difficulties. In the long-term, the costs associated with complications resulting from uncontrolled diabetes, such as renal failure and amputation, are very high. Approximately one-third of people with diabetes have psychological and/or social problems that impede their ability to self-manage their diabetes. ${ }^{1}$

\section{Epidemiology}

The evidence base for the interrelationship of diabetes with mental illness has increased over the past 13 years. In 2001, Anderson et al ${ }^{2}$ conducted a meta-analysis which indicated that the presence of diabetes doubled the risk of comorbid depression. A systematic review of more recent literature, published in 2012 by Roy and Lloyd, ${ }^{3}$ found rates of depression in people with type 1 and type 2 diabetes three times and twice those in the general population, respectively. An understanding of the bi-directional pathophysiological relationship between diabetes and depression has been elucidated by Rustad et al, ${ }^{4}$ but key posited aetiologies are activation of the innate immune system and increased activity of the hypothalamic - pituitary - axis, in addition to the psychological burden of the illness. The significance of a double diagnosis of diabetes with depression is illustrated by the associations with non-adherence to treatment, poor glycaemic control and increased numbers of complications (including diabetic retinopathy, nephropathy, neuropathy, macrovascular problems and sexual dysfunction). ${ }^{5-7}$ In addition, Park et al ${ }^{8}$ found a hazard ratio of 1.5 (1.35-1.66) for all-cause mortality in depressed patients with diabetes.

Eating disorders are also associated with diabetes. In a meta-analysis published in 2005, Mannucci et al ${ }^{9}$ found that bulimia nervosa is more prevalent in females with type 1 diabetes in comparison to the normal (ie non type 1) female population (1.73 vs 0.69 ). Evidence for eating disorders in 